\title{
Exploiting statistical independence for quantitative photoacoustic tomography
}

Lu An

Teedah Saratoon

Martina Fonseca

Robert Ellwood

Ben Cox 


\title{
Exploiting statistical independence for quantitative photoacoustic tomography
}

\author{
Lu An, Teedah Saratoon, Martina Fonseca, Robert Ellwood, and Ben Cox \\ Department of Medical Physics and Biomedical Engineering, University College London, \\ Gower Street, WC1E 6BT, UK
}

\begin{abstract}
To unlock the full capability of photoacoustic tomography as a quantitative, high resolution, molecular imaging modality, the problem of quantitative photoacoustic tomography must be solved. The aim in this is to extract clinically relevant functional information from photoacoustic images by finding the concentrations of the chromophores in the tissue. This is a challenging task due to the effect of the unknown but spatially and spectrally varying light fluence within the tissue. Many inversion schemes that include a model of the fluence have been proposed, but these have yet to make an impact in pre-clinical or clinical imaging. In this study, the statistical independence of the chromophore's distributions is proposed as a means of improving the robustness and hence the usefulness of the model-based inversion methods. This was achieved by minimising the mutual information between the estimated chromophore distributions in addition to the least squares data error within a gradient-based optimisation scheme. By applying the proposed inversion scheme to simulated multiwavelength photoacoustic images, it was shown that more accurate estimates for the concentrations of independent chromophores could be obtained in the presence of errors in the model parameters.
\end{abstract}

Keywords: Quantitative photoacoustic tomography, statistical independence, model-based inversion, mutual information, gradient-based minimisation, experimental uncertainty, multiwavelength

\section{INTRODUCTION}

Quantitative photoacoustic tomography aims to estimate how the chromophores are distributed (their concentration as a function of position) and hence provide functional information about the tissue. As an example, by applying spectroscopic analysis to fluence-corrected multiwavelength photoacoustic images, the concentrations of oxy- and deoxyhaemoglobin can potentially be estimated, which gives a measure of the blood oxygenation. In addition to quantifying endogenous chromophores, another important application of quantitative photoacoustic tomography is to detect and quantify the local accumulation of exogenous contrast agents and genetically encoded probes, which can be an useful tool for studying cellular or molecular processes in disease development.

Certain types of exogenous probes can be expected to be statistically independent from other prominent chromophores in the tissue. For example, fluorescent dyes can be targeted to disease-specific receptors whose spatial distribution is likely to be independent from the background tissue and blood. The statistically independent contrast agents can be spectrally unmixed using independent component analysis (ICA). ICA ${ }^{1}$ is a linear method for decomposing multiwavelength photoacoustic images into the independent source components, and it has been shown to provide more accurate unmixing than linear inversion. ${ }^{2}$ The disadvantages of ICA are that it cannot estimate the absolute values of the chromophores and, since it is based on a linear model, it does not account for the spectral colouring due to the light fluence. Model-based inversion methods ${ }^{3-5}$ overcome these limitations by incorporating a model of the fluence distribution. However, model-based inversion schemes are sensitive to inaccuracies in the model parameters, such as the light source position and absorption spectra of the chromophores, and therefore may lead to errors in the presence of experimental uncertainties. The aim of this study is to reduce these errors by including the statistical independence of the chromophores as additional information in the inversion.

Photons Plus Ultrasound: Imaging and Sensing 2017, edited by Alexander A. Oraevsky, Lihong V. Wang, Proc. of SPIE Vol. 10064, 1006419 · @ 2017 SPIE · CCC code: 1605-7422/17/\$18 · doi: 10.1117/12.2250290 


\section{ESTIMATING THE STATISTICAL INDEPENDENCE}

Statistical independence is defined based on probability densities. Two continuous random variables $\mathbf{y}_{1}$ and $\mathbf{y}_{2}$ are statistically independent if their joint probability density function (PDF), $\rho_{\mathbf{y}_{1}, \mathbf{y}_{2}}\left(y_{1}, y_{2}\right)$, can be factorised into their marginal PDFs, $\rho_{\mathbf{y}_{1}}\left(y_{1}\right)$ and $\rho_{\mathbf{y}_{2}}\left(y_{2}\right),{ }^{6}$ so that

$$
\rho_{\mathbf{y}_{1}, \mathbf{y}_{2}}\left(y_{1}, y_{2}\right)=\rho_{\mathbf{y}_{1}}\left(y_{1}\right) \rho_{\mathbf{y}_{2}}\left(y_{2}\right),
$$

where $y_{1}$ and $y_{2}$ denote possible outcomes of $\mathbf{y}_{1}$ and $\mathbf{y}_{2}$. The statistical independence between two variables can be measured using mutual information (MI). The MI estimates the amount of information one random variable contains about another random variable. If the variables are completely independent, they will not provide any information on each other, hence their MI will be zero. In quantitative photoacoustic tomography, this requires that knowing the concentration of one chromophore at a location does not reduce the uncertainty of the value for the concentration of the other chromophore at the same location. The mutual information $I$ between $\mathbf{y}_{1}$ and $\mathbf{y}_{2}$ is defined $\operatorname{as}^{6}$

$$
I\left(\mathbf{y}_{1}, \mathbf{y}_{2}\right)=\mathcal{H}\left(\mathbf{y}_{1}\right)+\mathcal{H}\left(\mathbf{y}_{2}\right)-\mathcal{H}\left(\mathbf{y}_{1}, \mathbf{y}_{2}\right),
$$

where $\mathcal{H}\left(\mathbf{y}_{k}\right)$ and $\mathcal{H}\left(\mathbf{y}_{1}, \mathbf{y}_{2}\right)$ are the entropy and the joint entropy of $\mathbf{y}_{1}$ and $\mathbf{y}_{2}$ respectively. They are defined by

$$
\mathcal{H}\left(\mathbf{y}_{k}\right)=-\int_{y_{k}} \rho_{\mathbf{y}_{k}}\left(y_{k}\right) \log _{2} \rho_{\mathbf{y}_{k}}\left(y_{k}\right) \mathrm{d} y_{k}
$$

and

$$
\mathcal{H}\left(\mathbf{y}_{1}, \mathbf{y}_{2}\right)=-\int_{y_{1}} \int_{y_{2}} \rho_{\mathbf{y}_{1}, \mathbf{y}_{2}}\left(y_{1}, y_{2}\right) \log _{2} \rho_{\mathbf{y}_{1}, \mathbf{y}_{2}}\left(y_{1}, y_{2}\right) \mathrm{d} y_{1} \mathrm{~d} y_{2} .
$$

Equation (2) can also be extended to define the MI between multiple random variables:

$$
I\left(\mathbf{y}_{1}, \ldots, \mathbf{y}_{K}\right)=\sum_{k=1}^{K} \mathcal{H}\left(\mathbf{y}_{k}\right)-\mathcal{H}\left(\mathbf{y}_{1}, \ldots, \mathbf{y}_{K}\right) .
$$

To calculate the MI between multiple chromophore concentrations $\mathbf{c}_{1}, \ldots, \mathbf{c}_{K}$, we consider them as random variables with the PDFs $\rho_{\mathbf{c}_{1}}, \ldots, \rho_{\mathbf{c}_{K}}$. The values of the concentration of the $k$ th chromophore $\mathbf{c}_{k}$ at different voxels, $\left[c_{k}^{(1)}, \ldots, c_{k}^{(M)}\right]$, are considered as data points or "observations" of the random variable. The total number of observations is equal to the number of voxels $M$. The PDF and the joint PDF can be estimated using the kernel density estimator, ${ }^{7}$ given by

$$
\rho_{\mathbf{c}_{k}}\left(\xi_{k}\right)=\frac{1}{M} \sum_{m=1}^{M} \kappa\left(\xi_{k}-c_{k}^{(m)}\right)
$$

and

$$
\rho_{\mathbf{c}_{1}, \ldots, \mathbf{c}_{K}}\left(\xi_{1}, \ldots, \xi_{K}\right)=\frac{1}{M} \sum_{m=1}^{M} \prod_{k=1}^{K} \kappa\left(\xi_{k}-c_{k}^{(m)}\right),
$$

where $\xi_{k}$ is the value at which the PDF is estimated and $\kappa(\cdot)$ is the kernel function. The key advantage of the kernel density estimator is that provided that a continuous and differentiable kernel is chosen, the estimated PDFs will also have those same desirable properties. ${ }^{7}$ Therefore, a Gaussian kernel given by

$$
\kappa(x)=\frac{1}{h \sqrt{2 \pi}} \exp \left(-x^{2} / 2 h^{2}\right)
$$

is used in this study, where $h$ is the kernel width, which determines the amount of smoothing in the estimations. By substituting Eqs. (6) and (7) into Eqs. (3), (4) and (5), the MI can be evaluated for the chromophores. 


\section{MODEL-BASED INVERSION WITH STATISTICAL INDEPENDENCE}

The initial pressure distribution, $p$, is a product of the Grüneisen parameter $\Gamma$, the absorption coefficient $\mu_{a}$, and the spatially and spectrally varying fluence $\phi$, which itself depends on the unknown chromophore concentrations. The initial pressure rise at location $\mathbf{r}$ and wavelength $\lambda$ can be expressed as

$$
p(\mathbf{r}, \lambda)=\Gamma(\mathbf{r}) \phi(\mathbf{r}, \lambda) \mu_{a}(\mathbf{r}, \lambda),
$$

where the absorption coefficient is given by $\mu_{a}(\mathbf{r}, \lambda)=\sum_{k} \alpha_{k}(\lambda) c_{k}(\mathbf{r})$, and $\alpha_{k}$ is the specific absorption coefficient of the $k$ th chromophore.

The model-based inversion scheme used in this study involves first making an initial guess for the unknown chromophore concentrations. Then, the fluence is modelled using the diffusion approximation to the radiative transfer equation based on the distribution of the absorption coefficient, which is calculated for the initial guess of the chromophore concentrations, and the scattering coefficient, which is assumed to be known. Using the modelled fluence, the initial pressure rise can be predicted using Eqn. (9), assuming that the Grüneisen parameter is known. The difference between the modelled and the measured initial pressure, $p^{\text {model }}$ and $p^{\text {meas }}$, at multiple wavelengths is defined as the data error $\varepsilon_{d}$,

$$
\varepsilon_{d}\left(\mathbf{c}_{1}, \ldots, \mathbf{c}_{K}\right)=\sum_{n=1}^{N} \sum_{m=1}^{M}\left[p_{m, \lambda_{n}}^{\text {model }}\left(\mathbf{c}_{1}, \ldots, \mathbf{c}_{K}\right)-p_{m, \lambda_{n}}^{\text {meas }}\right]^{2},
$$

where $N, M$ and $K$ denote the total number of wavelengths, voxels and unknown chromophores respectively. Estimates for the chromophore concentrations are found by iteratively adjusting their values until $\varepsilon_{d}$ is minimised.

In the idealised case, an accurate recovery of the chromophore concentrations can be obtained using the model-based inversion method, provided $N$ is larger or equal to $K$. However, in practice, modelling error or noise in the data may lead to the minimum of the data error occurring at the wrong solution, which would result in inaccurate estimations of the chromophore concentrations. The statistical independence, on the other hand, is an intrinsic property of the chromophores. Therefore, the MI between the chromophore concentrations is not affected by errors in the forward model, and a minimum will always occur at the true solution for independent chromophores. Hence, by incorporating the MI as an additional term in the error functional, such that

$$
\varepsilon_{d+M I}\left(\mathbf{c}_{1}, \ldots, \mathbf{c}_{K}\right)=\sum_{n=1}^{N} \sum_{m=1}^{M}\left[p_{m, \lambda_{n}}^{\text {model }}\left(\mathbf{c}_{1}, \ldots, \mathbf{c}_{K}\right)-p_{m, \lambda_{n}}^{\text {meas }}\right]^{2}+\gamma I\left(\mathbf{c}_{1}, \ldots, \mathbf{c}_{K}\right),
$$

where $\gamma$ denotes the weight parameter for the MI term, more accurate solutions could potentially be obtained.

\section{THE NUMERICAL PHANTOM}

A 2D numerical phantom was used to investigate the effect of including the MI term in the error functional. The phantom represents a $5 \times 5 \mathrm{~mm}$ region with an element spacing of $100 \mu \mathrm{m}$. As shown in Fig. 1, it contains six insertions arranged in two columns, which mimic regions in the tissue containing various absorbers with different concentrations. The insertions in the left column contain copper chloride of concentrations 12,24 and $36 \mathrm{~g} / \mathrm{l}$ in increasing order where the top insertion has the lowest concentration. The right column of insertions contains nickel chloride of concentrations 133, 266 and 399g/l, also in increasing order from the top insertion. These concentrations give rise to an average absorption of $0.6 \mathrm{~mm}^{-1}$ at the insertions at $790 \mathrm{~nm}$ for both columns. The spatial arrangements of the copper chloride and the nickel chloride are such that they are statistically independent of each other. The whole domain contains water and the background region outside the insertions also contains India ink such that its absorption is similar to that of tissue. These contrast agents are chosen to mimic a planned experimental phantom measurement. The specific absorption coefficients of copper chloride and nickel chloride are shown in Fig. 2(a) and the absorption coefficients of India ink and water are shown in Fig. 2(b). The reduced scattering coefficient is spatially homogeneous in the phantom and its wavelength dependence is shown in Fig. 2(c). 


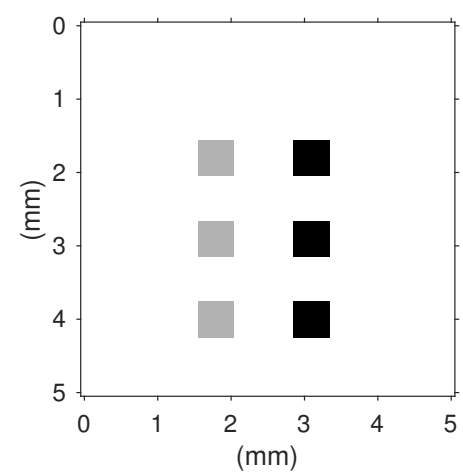

Figure 1. The structure of the numerical phantom. The grey and black regions indicate the insertions containing copper chloride or nickel chloride respectively, and the white region indicates the background area.

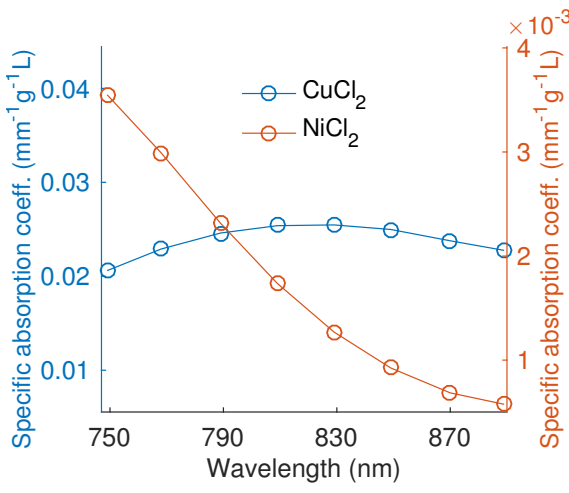

(a)

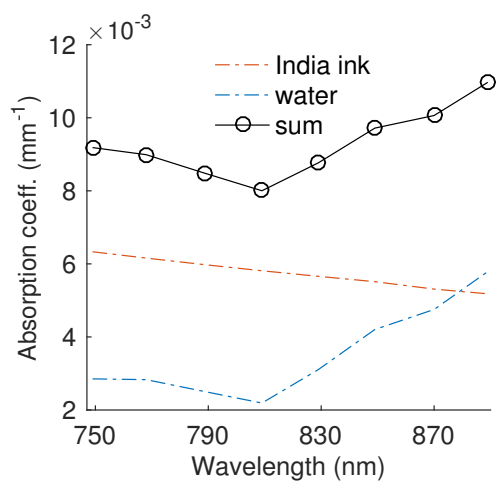

(b)

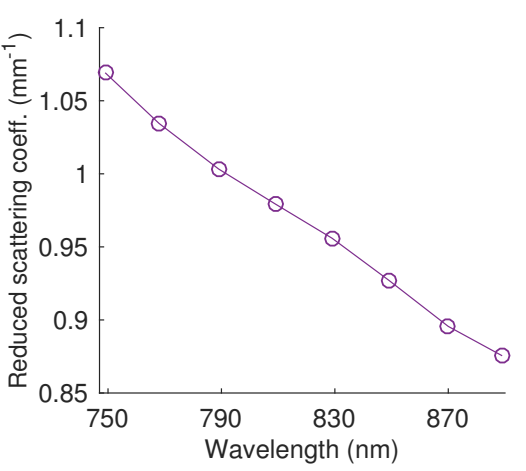

(c)

Figure 2. (a) The specific absorption spectra of copper chloride and nickel chloride. (b) The absorption spectra of India ink and water. ${ }^{8}$ The sum of their absorption is indicated in the black curve. (c) The wavelength dependent reduced scattering coefficient, which is based on the reduced scattering coefficient of $1 \%$ Intralipid. ${ }^{9}$ The background absorption and scattering amplitude are comparable to that of generic biological tissue for the same wavelength range. ${ }^{10}$ The spectra of copper chloride, nickel chloride and India ink are based on transmission measurements using a spectrophotometer (Lambda 750S, Perkin Elmer).

The fluence is modelled using the diffusion approximation ${ }^{11}$ for 8 wavelengths with equal spacing between 750 and 890nm, assuming that the light source is a Gaussian beam with a width of $3 \mathrm{~mm}$ at the top of the domain. The Grüneisen parameter is assumed to be spatially constant and the acoustic reconstruction is assumed to be perfect, such that the reconstructed initial pressure rise is equal to the product of the fluence and the absorption coefficient. A Gaussian noise with magnitude equal to $10 \%$ of the mean initial pressure is added to the simulated images.

\section{INVERTING FOR THE CHROMOPHORE CONCENTRATIONS}

To simulate realistic imaging scenarios, in which some of the model parameters may contain experimental error, three case studies were conducted. In each case, errors were added to different model parameters in the inversion: In case A, a $10 \%$ random error was added to the absorption coefficients. In case B, the beam diameter was set to be $75 \%$ larger or smaller than the true value in steps of $25 \%$. In case C, errors up to $\pm 75 \%$ were added to the scattering amplitude, also in steps of $25 \%$.

The limited memory BFGS algorithm ${ }^{12}$ was used to minimise the error functional in Eqs. (10) or (11), where the unknown parameters were the concentrations of copper chloride, nickel chloride, India ink and water at each pixel, and the MI was evaluated between the copper chloride and the nickel chloride. The algorithm searches for the minimum of the error functional using the gradients of the data error and the MI term, which can be found in Cox et $a l^{5}$ and Panagiotou et al. ${ }^{13}$ The computational time required for evaluating the gradient of the 
MI term was significantly reduced by utilising the fact that Eqs. (6) and (7) have convolution structures and therefore can be calculated efficiently using fast Fourier transforms. ${ }^{14}$

To investigate the effect of incorporating the MI term, the minimisation algorithm was run for 300 iterations both using $\varepsilon_{d}$ and $\varepsilon_{d+M I}$ for each scenario. In order to prevent the algorithm from being trapped in the local minima of the MI before reaching the vicinity of the true solution, the weight parameter of the MI term was set to be zero for the first 200 iterations and 20 for the remaining iterations for all inversions using $\varepsilon_{d+M I}$.

The estimated and the true concentrations of copper chloride and nickel chloride for case A are shown in Fig. 3. The left column shows the estimations from the inversion where only the data error term is minimised, while the middle column shows the results using the error functional including the MI. By comparing these to the true concentrations shown in the right column, one can see that the errors are smaller for the inversion using $\varepsilon_{d+M I}$ than $\varepsilon_{d}$. Most prominently, the cross-talk error in the nickel chloride estimation is significantly reduced with $\varepsilon_{d+M I}$. The average error of the absolute concentrations at the insertions is $4 \%$ for the inversion using $\varepsilon_{d}$, and it is reduced to $1 \%$ when $\varepsilon_{d+M I}$ is used.
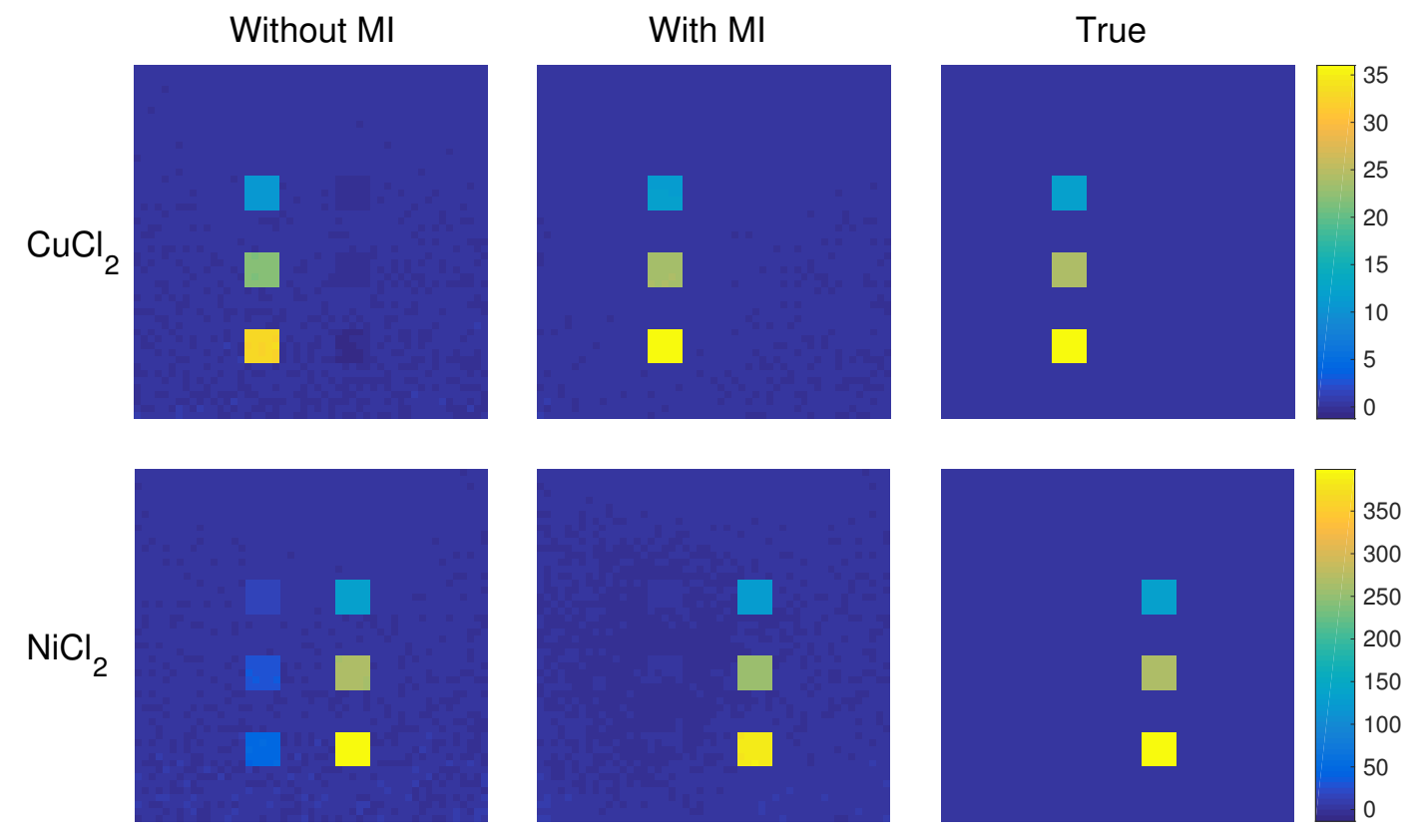

Figure 3. The estimated chromophore concentrations of copper chloride (top row) and nickel chloride (bottom row) from the inversion without the MI term (left column) and with the MI term (middle column), and the true concentrations (right column). The crosstalk error seen in the estimated nickel chloride concentration in the left column is significantly reduced when MI is used.

Figure 4(a) shows the average errors of the estimated chromophore concentrations at the insertions as a function of changes in the beam diameter from the true value (case B). The blue and red curves represent the estimations from the inversions using $\varepsilon_{d}$ and $\varepsilon_{d+M I}$ respectively. The results show that, as expected, the concentration errors increase as the inaccuracy in the beam diameter increases. However, the errors are smaller when $\varepsilon_{d+M I}$ is minimised compared to $\varepsilon_{d}$ for all data points. The same trends are seen when the scattering amplitude is varied (case C), as shown in Fig. 4(b). The errors are generally larger in case C than case B, but including the MI is nonetheless consistently providing more accurate estimates compared to only using the standard data error. Overall, the average relative improvement in accuracy using $\varepsilon_{d+M I}$ compared to $\varepsilon_{d}$ for all cases is $24 \%$.

\section{SUMMARY AND DISCUSSION}

This study demonstrated that the statistical independence between chromophores can be exploited in modelbased inversion methods to reduce the errors of the estimated chromophore concentrations. It was shown that 


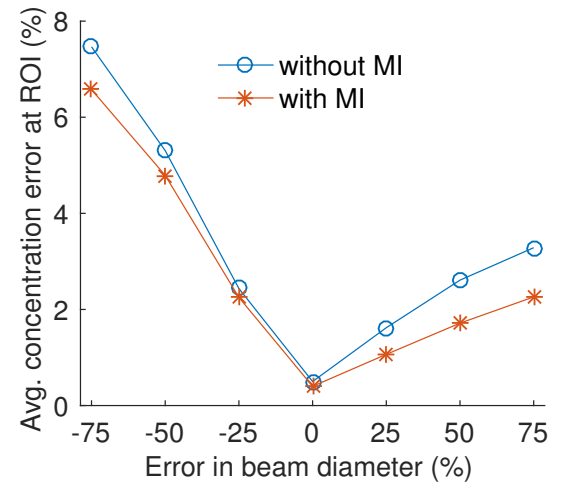

(a)

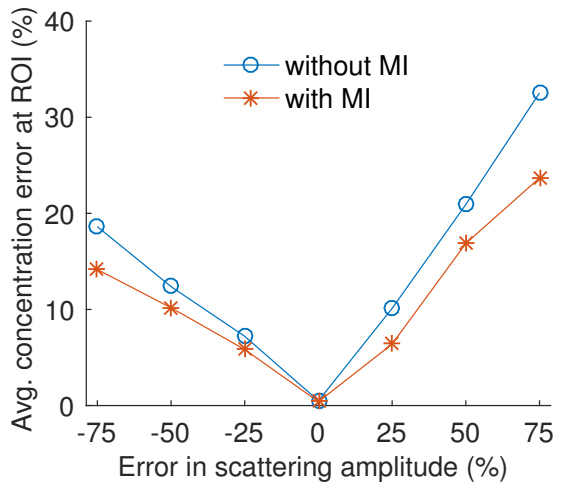

(b)

Figure 4. The average errors of the estimated chromophore concentrations at the insertions as a function of errors in (a) the beam diameter and (b) the scattering amplitude. The errors from the inversion with the MI term (red curve) is consistently smaller than the errors from the standard inversion without using MI (blue curve).

by including a MI term in the error functional, more accurate quantification can be achieved in the presence of errors in the model parameters, assuming that the chromophores are known to be statistically independent $a$ priori.

Similar to other regularisation methods, an appropriate weight parameter for the MI term is required for the inversion to result in accurate quantification. In this study, the weight was determined using manual trial and error. However, for in vivo applications, where the true solution is unknown, it will be necessary to develop a programmatic method for finding the optimal weight parameter.

In the simulated case studies presented in this paper, inaccuracies were added to one model parameter at the time. However, in practical measurements, there may be a combination of different sources of errors, resulting in larger model mismatch. The model inaccuracies may be especially severe in in vivo experiments where some model parameters may be difficult to control or measure, which makes the application of model-based inversion in experimental preclinical or clinical imaging studies challenging. By incorporating the additional information of statistical independence, however, it may become more feasible to use model-based inversion for in vivo images. This could potentially enable more accurate quantification of the accumulation of independent probes in imaging studies using exogenous contrast agents or genetic reporters.

\section{REFERENCES}

[1] Hyvärinen, A. and Oja, E., "Independent component analysis: algorithms and applications," Neural Netw. 13(4-5), 411-430 (2000).

[2] Glatz, J., Deliolanis, N. C., Buehler, A., Razansky, D., and Ntziachristos, V., "Blind source unmixing in multi-spectral optoacoustic tomography," Opt. Express 19(4), 3175-3184 (2011).

[3] Saratoon, T., Tarvainen, T., Cox, B., and Arridge, S., "A gradient-based method for quantitative photoacoustic tomography using the radiative transfer equation," Inv. Prob. 29(7), 075006 (2013).

[4] Bal, G. and Ren, K., "On multi-spectral quantitative photoacoustic tomography in diffusive regime," Inverse Probl. 28(2), 025010 (2012).

[5] Cox, B. T., Arridge, S. R., and Beard, P. C., "Estimating chromophore distributions from multiwavelength photoacoustic images," J. Opt. Soc. Am. A 26(2), 443-455 (2009).

[6] Hyvärinen, A., Karhunen, J., and Oja, E., [Independent Component Analysis], John Wiley \& Sons, Toronto (2004).

[7] Silverman, B. W., [Density estimation for statistics and data analysis], Chapman and Hall, London (1986).

[8] Kou, L., Labrie, D., and Chylek, P., "Refractive indices of water and ice in the 0.65-to 2.5- $\mu$ m spectral range," Appl. Opt. 32(19), 3531-3540 (1993). 
[9] Van Staveren, H. J., Moes, C. J., van Marie, J., Prahl, S. A., and Van Gemert, M. J., "Light scattering in lntralipid-10\% in the wavelength range of 400-1100 nm," Appl. Opt. 30(31), 4507-4514 (1991).

[10] Jacques, S. L., "Optical properties of biological tissues: a review," Phys. Med. Biol. 58(11), R37-R61 (2013).

[11] Schweiger, M. and Arridge, S., "The Toast++ software suite for forward and inverse modeling in optical tomography," J. Biomed. Opt. 19(4), 040801 (2014).

[12] Nocedal, J. and Wright, S., [Numerical Optimization], Springer Science \& Business Media, New York (2006).

[13] Panagiotou, C., Somayajula, S., Gibson, A. P., Schweiger, M., Leahy, R. M., and Arridge, S. R., "Information theoretic regularization in diffuse optical tomography," J. Opt. Soc. Am. 26(5), 1277-1290 (2009).

[14] Shwartz, S., Zibulevsky, M., and Schechner, Y. Y., "ICA using kernel entropy estimation with NlogN complexity," in [Independent Component Analysis and Blind Signal Separation: Fifth International Conference, ICA 2004], Puntonet, C. G. and Prieto, A., eds., Lecture Notes in Computer Science 3195, Springer Berlin Heidelberg, Berlin (2004). 